\title{
A Study on Automobile Form Design Support Method "AFD-SM"
}

Hiroki Asami, Aoyama Gakuin University, Japan

Takashi Ando, Aoyama Gakuin University, Japan

Manabu Yamaji, Aoyama Gakuin University, Japan

Kakuro Amasaka, Aoyama Gakuin University, Japan

\begin{abstract}
In this research, by assigning attention to the automobile "form," details that were previously ambiguous, such as "roundness" and "angle", are represented numerically. With the aim of measuring the degree of influence of customer sensibility, the "Automobile Design Form Support Method (ADF-SM)" is utilized. Using CAD, the authors have constructed a model of a formaltered automobile to quantitatively evaluate the degree of influence of customer sensitivity. By using this support method, an automobile form is created, which expresses the concept sought by the customer, verifying its effectiveness.
\end{abstract}

Keywords: Automobile, ADF-SM, Reverse Engineering, 3D-Design CAD

\section{INTRODUCTION}

n this research, we aim to further the development of automobile design concept support research by
giving attention to the automobile "form," a domain of high novelty. Details that were formerly
ambiguous, such as "roundness" and "angle", are quantified with the aim of effectively supporting design
and development by relating to customer sensitivity.

\section{BACKGROUND}

In order to develop and offer appealing, customer-oriented products, it is important to incorporate customer feelings and words into the new product development process and to fully put into practice the concept of "Customer Science." These days, changes in customer preferences toward automobiles are very pronounced and are becoming increasingly diversified and complicated. In order to build functionally into durable goods, it is becoming important to use "sensitive engineering" that both quantifies and grasps the images and feelings in customers" hearts, such as their favorite foods.

Regarding automobile design, it is very important to use statistical methods to develop a commodity which precisely answers customer demands. Accordingly, various research studies have adopted this approach using "Design SQC" and the "profile design support tool." Generally, customers notice three elements of the design: (1) profile design (proportions), which is noticed at long-to-mid distance, (2) form, which is noticed at mid-to short distance, and (3) surface, which is noticed at short distance to close range.

Regarding proportion, research to clarify the relationship between form and customer sensitivity has previously been undertaken from various points of view. Furthermore, Ando et al connected with customer sensitivity by paying attention to the modeling form of the front fender circumference and numerically evaluating "roundness" and "angle" via CAD. However, research findings regarding the basic actions taken when a customer looks at a car indicate that the slant viewpoint taken from the car front is also important.

Accordingly, this research expands upon the frontal view taken in previous research, by paying attention to the change of form accompanying a side view, which occurs when a customer looks at a car from an angle (i.e., at a 
slant from the front), measuring the degree of influence of customer sensitivity to the form change and reflecting this information in the design.

\section{NECESSITY OF NUMERICAL FORM REPRESENTATION BY CAD}

In order to visualize the degree of influence of customer sensitivity due to the change of the form, digitalization of the form is indispensable. In this research, the CAD (Computer Aided Design) software is utilized as an expedient for form digitalization. The advantages of using CAD include: (1) "form digitalization", in which a numerical definition of the form is created (parametrization), is possible using CAD; (2) since the front, side, rear, etc. of the automobile are verifiable from all angles, establishing a relationship with customer sensitivity is possible; (3) it may be noted that, as in the present design process, the use of CAD as a tool for expressing form for modeling, etc. is mainstream use. Further, in this research, CATIA V5 is used as a CAD software.

Furthermore, reverse engineering was used to increase the accuracy of the CAD model. This reverse engineering involved the measurement of a clay model made as a advance conceptualization of the product, with data regarding product form characterizing this actual object used as a basis for creating CAD data. In this research, the 3D picture measurement software, "QM-3000", produced by TOPCON Techno House Corporation, was used. By non-contact measurement, it was possible to convent in 3D the delicate curved line of the design, which cannot be expressed by dimension.

\section{CONSTRUCTION OF THE AUTOMOBILE DESIGN FORM SUPPORT METHOD (ADF-SM)}

As mentioned above, it is an important research task to evaluate the "roundness" and "angle" of an automobile form, which have remained ambiguous until now, and to relate these quantities to customer sensitivity in our primary theme - development of an automobile design conception support method.

Thus, establishment of the "Automobile Design Form Support Method (ADF-SM)", shown in Figure 1 for business solution, is undertaken. In STEP 1, in order to increase the accuracy of the CAD model, a CAD model design, which utilizes reverse engineering, is performed. In STEP 2, a sensitivity evaluation sheet, which uses nine respective form types for the front side, is created, and data collection to determine customer feelings is performed using the resulting sheet.

In STEP 3, the resulting data is used to quantitatively grasp the relationship between changes in form and the resulting customer feelings. In STEP 4, by connecting the data collected in STEP 3 regarding the front and the side areas, an analysis is made concerning the related characteristics of the whole (front and side.) Furthermore, a new model is created from the analysis result and verification by an additional questionnaire appraisal performed.

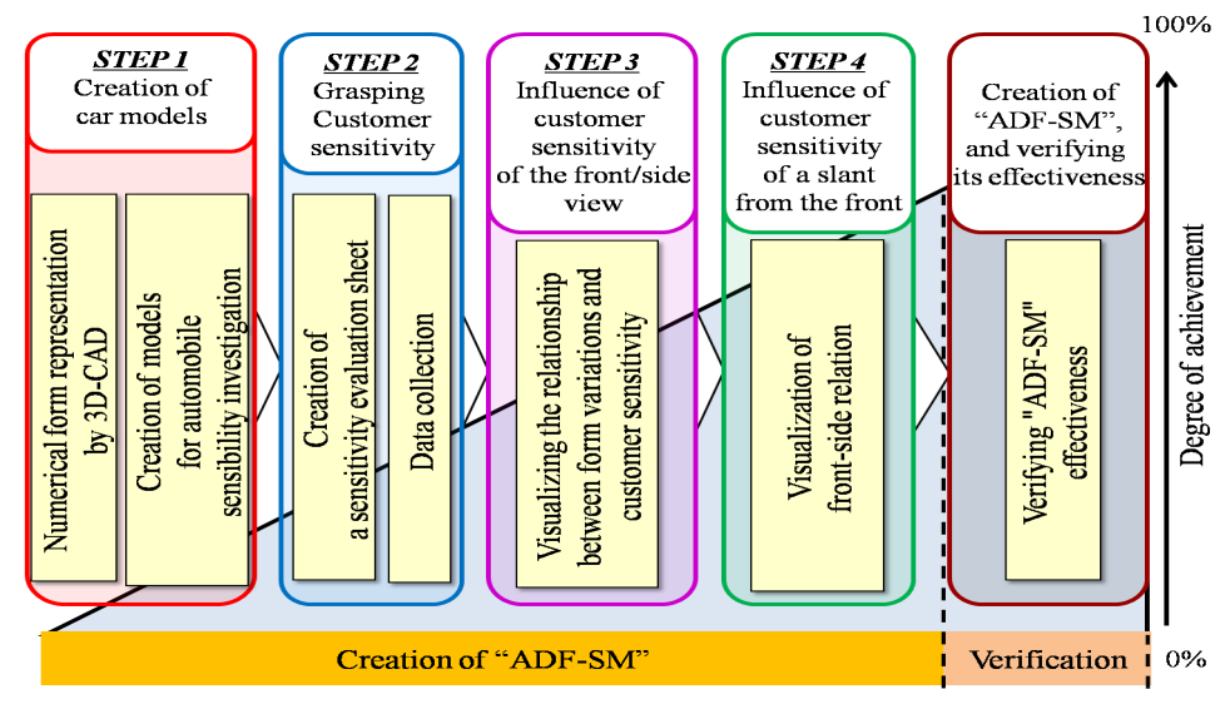

Figure 1: Automobile Design Form Support Method "ADF-SM" 
Table 1: Form Shape Parameters for Nine Car Model Types (Side View)

\begin{tabular}{|c|c|c|c|}
\hline Car type/Form shape & Angle of the front pillar $\left(^{\circ}\right)$ & Angle of the belt line $\left({ }^{\circ}\right)$ & Angle of the character line $\left({ }^{\circ}\right)$ \\
\hline TypeA & 45 & 2 & 25 \\
\hline TypeB & 30 & 0 & 15 \\
\hline TypeC & 30 & 0 & 55 \\
\hline TypeD & 30 & 5 & 15 \\
\hline TypeE & 30 & 5 & 55 \\
\hline TypeF & 60 & 0 & 15 \\
\hline TypeG & 60 & 0 & 55 \\
\hline TypeH & 60 & 5 & 15 \\
\hline TypeI & 60 & 5 & 55 \\
\hline
\end{tabular}
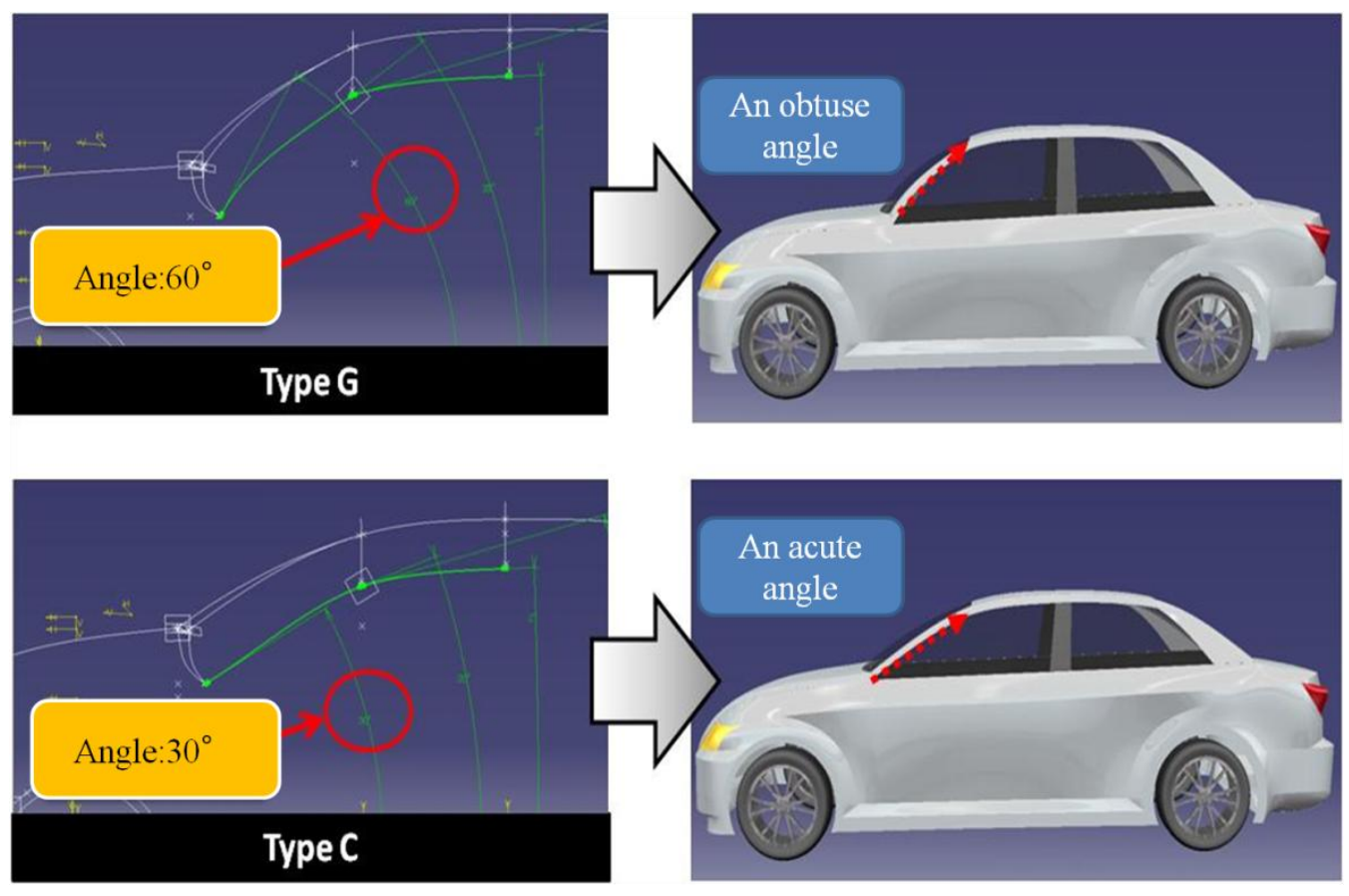

Figure 2: Example of A Change of Form (Angle of the Front Pillar)

\section{APPLICATION EXAMPLE}

Research findings regarding the basic actions taken when a customer is in front of an automobile clearly indicate that the main attention is on the front area. Thus, in order to investigate the influence of the front as part of the appearance design conception support method, the front and side are investigated independently and the relationship with customer sensitivity is visualized by investigating the mutual degree of influence.

\section{Creation of a Model for Automobile Sensibility Investigation - STEP 1}

As a means of associating customer sensitivity (as expressed through words) with form, a car model, including several types of determined parameters, is created by CAD, the meaning of which is clarified by performing a sensitivity evaluation. At that time, it is necessary to consider what kind of form change may be considered in the shape of each basic form. Then, according to the "character attachment of the automobile," a 
designer investigates the part to which the greatest importance has been attached.

For the front, the (1) bonnet edge, (2) bonnet, and (3) fender were used as factors, while the (4) front pillar, (5) belt line, and (6) character line were used as factors for the side, and the degree of changes in these form factors was regarded as a standard. In this research, the purpose was to intentionally vary the form so that the corresponding changes in customer impressions could be checked. Then a model for the investigation of nine car types (A-I) was created using three side regions of attention as factors.

The form shape parameters and corresponding customer impressions are shown in Table 1 for each car model type. Similarly, a model for investigating nine car types (Type A'-Type I') was created for the front. As a comparative example of a change of form, Figure 2 shows an example where the angle of the front pillar for Types $\mathrm{C}$ and $\mathrm{G}$ is used as the single axis of change.

\section{Grasping Customer Sensitivity - STEP 2}

The purpose of the sensitivity evaluation questionnaire is to explore the differences in the impressions felt by customers, regarding nine models in which the forms of the front and side have been changed. Accordingly, subjects are asked for questionnaire replies in the same form regarding all models. This data are analyzed to determine the relation between subject impressions and form. In addition, the candidates for investigation targeted the younger age group (the 20 's).

In the questionnaire, a seven-step evaluation using sensitivity words, is performed. In order to select suitable sensitivity words, a bibliographic search and an investigation hearing with the designer were conducted and 42 sensitivity words related automobile appearance were extracted. This set of words was then narrowed to those having a close relation, yielding the 12 sensitivity words shown in Table 2.

Table 2: 12 Sensitivity Words

\begin{tabular}{|c|c|c|}
\hline Sophisticated & Intellectual & Elegant \\
\hline Traditional & Advanced & High class \\
\hline Characteristic & Simple & Composed \\
\hline Cute & Powerful & Sporty \\
\hline
\end{tabular}

Visualizing the Relationship between Form Variations and Customer Sensitivity - STEP 3

Analysis is conducted at two steps: 5.3.1 form change and visualization of customer sensitivity, and 5.3.2 visualization of front/side relation.

\section{Determination of Shape Parameter Sensitivity Word Weights}

In order to clarify the weights of the sensitivity words for each shape parameter, a multiple regression analysis was conducted by taking sensitivity words and shape parameters as subject and explanatory variables, respectively. In this case, each standardized partial regression coefficient of the explanatory variable is designated as the weight for the subject variable. In this research, since 12 sensitivity words exist, 12 multiple linear regression analyses were performed by fixing the explanatory variable and changing the subject variable. 
Based on this, the weighted path diagram between the shape parameters and sensitivity words is created using the desired standard partial regression coefficients for the sensitivity words. The aim is, by using the model as an experiment, to be able to numerically read which kind of sensitivity feature the model serves as, and furthermore, to obtain the ability to distinguish which shape parameter should be changed to approach a target sensitivity feature of interest.

As an example, the shape parameter sensitivity path diagram is shown in Figure 3 from the standardized partial regression coefficient for "sophisticated." In this figure, the strength of the relationship for the shape parameter with strong weight is expressed by the thickness of the arrow, regardless of the positive/negative sign of the coefficient.

From the path diagram in Figure 3, it is seen that the angle of the front pillar exerts the strongest influence and an especially acute angle for the front pillar conveys an impression of "sophisticated." Moreover, the degree of influence each word could be visualized by conducting the same analysis for the other sensitivity words.

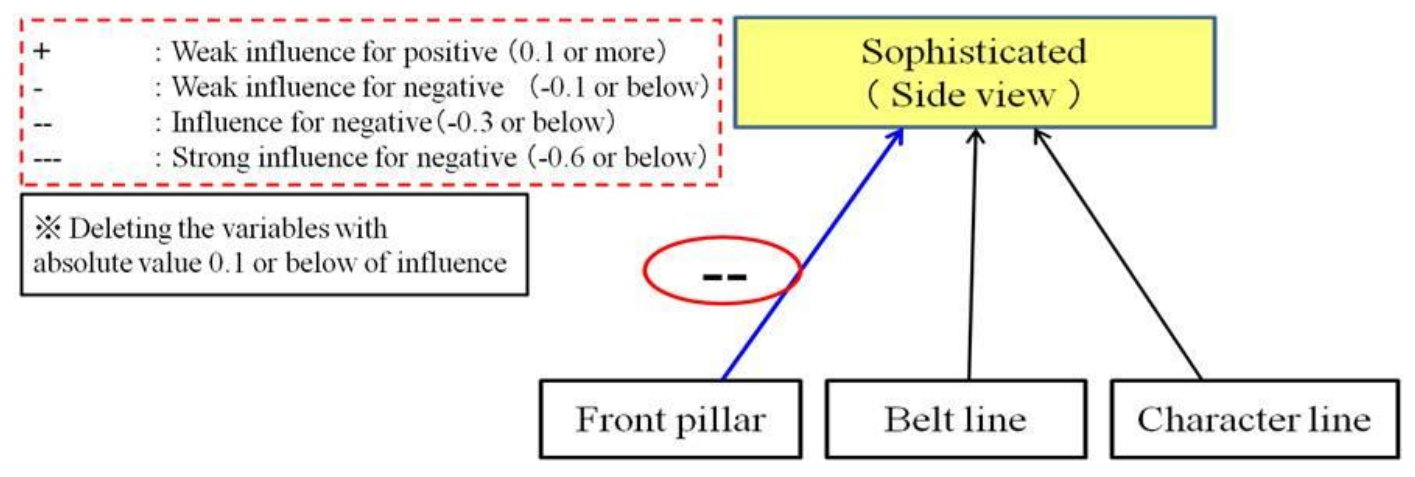

Figure 3 Shape Parameter Sensitivity Path Diagram for "Sophisticated" (Side View)

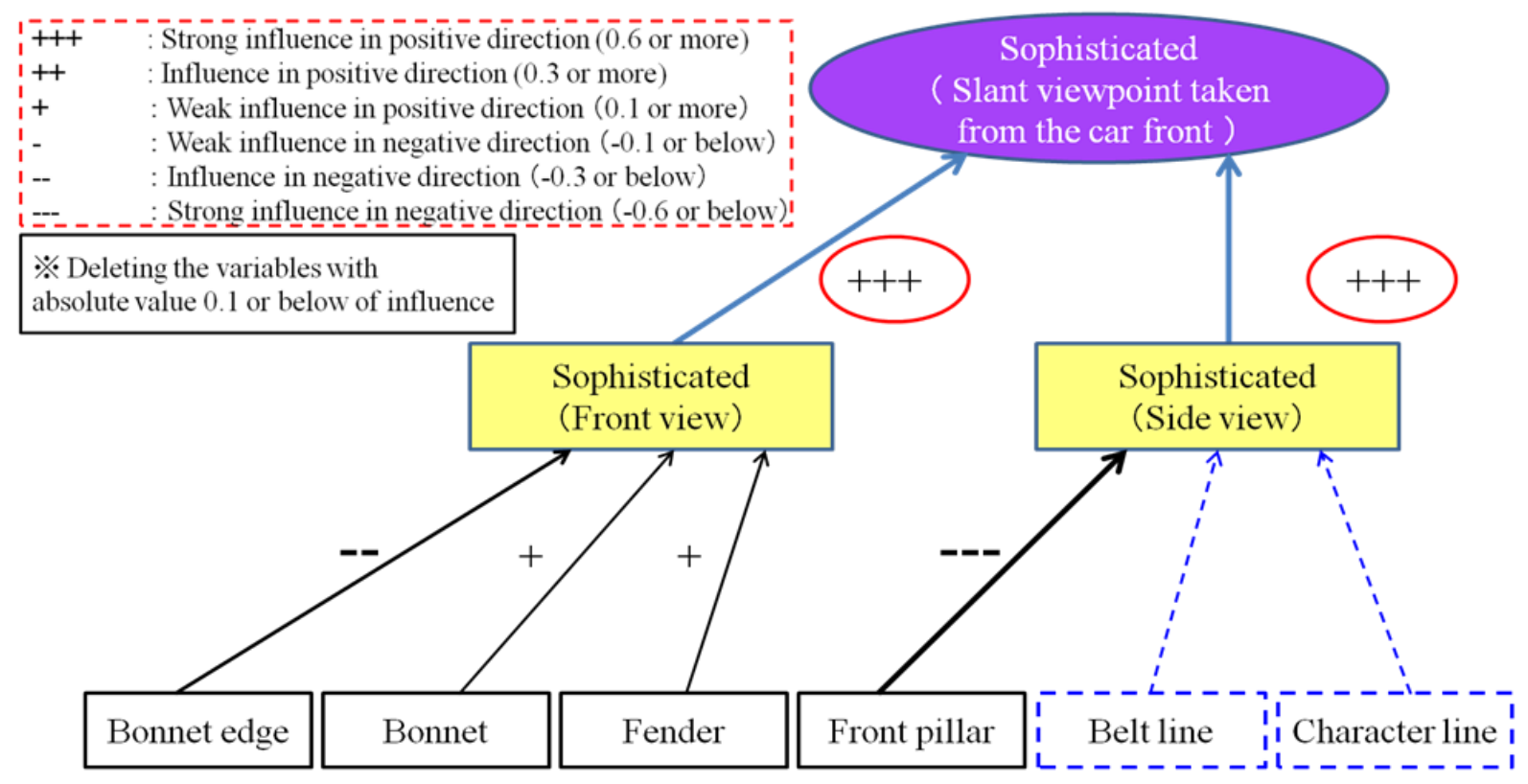

Figure 4: Path Diagram for "Sophisticated" Utilizing Covariance Structural Analysis 


\section{Visualization of the Front/Side of Relationship - STEP 4}

Covariance structural analysis was also conducted to obtain an overall sensitivity evaluation by combining separate sensitivity evaluations for a front part and a side part into a synthetic variable in order to examine their mutual influence. As an example, the path diagram for "sophisticated" is shown in Figure 4. Here, the model was improved by deleting the variables in 5.3.1 with very small degrees of influence, and the analysis was undertaken. From the path diagram in Figure 4, one front part and one side part each exert large degrees of influence and the angle of the front pillar conveys the sensitivity evaluation "sophisticated" as the shape parameter with the largest degree of influence. By conducting the same analysis for all sensitivity words, the front/side relationships can be comprehensively evaluated.

\section{VERIFICATION}

Based on the above information, it is clear that to the younger generation, the concept of "advanced", conveys a sense of stylishness. As a result, it is clear that an "advanced" image can be expressed via a specific combination of five points: (1) deep bonnet edge, (2) bonnet with swelling, (3) front pillar in an acute angle, (4) upward belt line, and (5) strengthened character line, each parameterized and materialized by CAD as a design. As a result of conducting a secondary questionnaire and analysis as verification of the new model, the expectation that it was possible to express the form, which conveys an impression of "advanced" to younger age group customers, was confirmed. Accordingly, the effectiveness of this research was considered to be verified.

\section{CONCLUSION}

A multiple regression analysis was undertaken to grasp the weight of the response of each sensitivity word to each shape parameter by determining the associated shape parameter-sensitivity word weights. By using each standardized partial regression coefficient of an explanatory variable as a subject variable weight, a path diagram, with weights between shape parameters and sensitivity words, was created. In this manner, the model was established on an experimental basis and it become possible to distinguish the types of sensitivity features the model serves to read numerically. Furthermore, when approaching a sensitivity feature, interpreted as an aim, it was possible to distinguish which shape parameter should be changed.

In addition, covariance structural analysis was applied to obtain a whole sensitivity evaluation, in order to comprehensively grasp the relationship of front and side regions to the 12 selected sensitivity words, by combining separate sensitivity evaluations of front and side parts into a synthetic variable.

\section{AUTHOR INFORMATION}

Hiroki Asami is a graduate student of the College of Science and Engineering at Aoyama Gakuin University.

Takashi Ando received his Master of Engineering degree from the College of Science and Engineering at Aoyama Gakuin University.

Manabu Yamaji is a researcher in the Advanced Research Center for Human Sciences at Waseda University, Japan. He received his Master of Engineering degree in Graduate School of Information Systems at University of ElectroCommunications in 1999. His current research and teaching interests are in the general area of production engineering. In particular, he is interested in Total Quality Management, Computer Aided Engineering.

Kakuro Amasaka is a Professor in the College of Science and Engineering at Aoyama Gakuin University, Japan. $\mathrm{He}$ received his Ph.D. degree in Precision Mechanical and System Engineering, Statistics and Quality Control at Hiroshima University in 1997. His current research and teaching interests are in the general area of production engineering. In particular, he is interested in New JIT. He is a member of POMS and EurOMA. 


\section{REFERENCES}

1. Amasaka, K. (2005), Constructing a Customer Science Application System "CS-CIANS" - Development of a Global Strategic Vehicle Lexus Utilizing New JIT-, WSEAS Transactions on Business and Economics, Issue3, Vol.2, pp.135-142.

2. Yamaji, M. and Amasaka, K. (2009), Intelligence Design Concept Method Utilizing Customer Science, The Open Industrial and Manufacturing Engineering Journal, Vol.2, pp.21-25.

3. Mori, N. (1996), Design Engineering, Asakura Syoten. (in Japanese)

4. Shinohara, A., Sakamoto, H. and Shimizu, Y. (1996), Invitation to Kansei Engineering, Morikita Shuppan. (in Japanese)

5. Amasaka, K. (2007), The Validity of "TDS-DTM", A Strategic Methodology of Merchandise - Development of New JIT, Key to the Excellence Design "LEXUS"-, The International Business \& Economics Research Journal, Vol.6, No.11, pp.105-115.

6. Okazaki, R., Suzuki, M. and Amasaka, K. (2000), Study on the Sense of Values by Age using Design SQC, The Japan Society for Production Management, The 11th Annual Technical Conference, pp. 139-142. (in Japanese)

7. Amasaka, K., Nagaya, A. and Shibata, W. (1999), Studies on Design SQC with the Application of Science SQC - Improving of Business Process Method for Automotive Profile Design, Japanese Journal of Sensory Evaluations, Vol.3, No.1, pp.21-29.

8. Amasaka, K. and Nagaya, A. (2002), Engineering of the New Sensitivity in the Vehicle, Development of the Articles over the Sensitivity, International Journal of Kansei Engineering, Nihon Shuppan Service Press, pp.55-72. (in Japanese)

9. Amasaka, K. (2004), Science SQC, New Quality Control Principle: The Quality Strategy of Toyota, Springer.

10. Aoyama, H. (2004), Digital style design based on "Kansei", Journal of the Japanese Society for Quality Control, Vol. 34, No.3, pp. 52-57, (in Japanese).

11. Li, Y., Kamaike, M. and Ueda, E. (2007), Grasp the Causal Relations between Design Elements of Passenger Car and Construction of Needs: Analysis of the Customers' Activities while Purchasing Passenger Cars in China, Bulletin of Japanese Society for Science of Design, Vol. 54, No.3, pp. 65-74. (in Japanese)

12. Masuyama, E. (1996), Measuring an Image to Appear on a Heart, Industry and Science Systems, (in Japanese).

13. $\quad$ Mori, N. (1996), Left brain Designing, Kaibundou Syuppan. (in Japanese)

14. Yanagisawa, H. and Fukuda, S. (2004), Development of Interactive Industrial Design Support System Considering Customer's Evaluation: Shape Design of Eyeglass Frame, JSME International Journal Series C: Dynamics Control Robotics Design \& Manufacturing, Vol.47, No. 2, pp. 762-769.

15. Ando, T., Yamaji, M. and Amasaka, K. (2008), A Study on Construction of Automobile Design Concept Support Methods - Visualization of Form and Customer Sensibility Utilizing Design CAD-, The 38th Annual Conference of The Japanese Society for Quality Control, pp.177-180. (in Japanese)

16. Yamaji, M., Murat, M., Hifumi, S. and Amasaka, K. (2010), Developing a Strategic Advertisement Method "VUCMIN" to Enhance the Desire of Customers for Visiting Dealers, Journal of Business Case Studies, Vol.6. No.3, pp.1-11. 


\section{NOTES}

